\title{
Orbital Origin of Extremely Anisotropic Superconducting Gap in Nematic Phase of FeSe Superconductor
}

Defa Liu, ${ }^{1,3}$ Cong Li ${ }^{1,2}$ Jianwei Huang,,${ }^{1,2}$ Bin Lei, ${ }^{4}$ Le Wang, ${ }^{1,2}$ Xianxin Wu, ${ }^{5}$ Bing Shen,,${ }^{1,2}$ Qiang Gao, ${ }^{1,2}$ Yuxiao Zhang, ${ }^{1}$ Xu Liu, ${ }^{1}$ Yong Hu, ${ }^{1,2}$ Yu Xu ${ }^{1,2}$ Aiji Liang, ${ }^{1}$ Jing Liu, ${ }^{1,2}$ Ping Ai, ${ }^{1,2}$ Lin Zhao, ${ }^{1}$ Shaolong He, ${ }^{1}$ Li Yu, ${ }^{1}$ Guodong Liu, ${ }^{1}$ Yiyuan Mao, ${ }_{1,2}$ Xiaoli Dong, ${ }^{1}$ Xiaowen Jia, ${ }^{6}$ Fengfeng Zhang, ${ }^{7}$ Shenjin Zhang, ${ }_{7}^{7}$ Feng Yang, ${ }^{7}$ Zhimin Wang, ${ }^{7}$ Qinjun Peng, ${ }^{7}$ Youguo Shi, ${ }^{1}$ Jiangping Hu, ${ }^{1,2,8}$ Tao Xiang, ${ }^{1,2,8}$ Xianhui Chen, ${ }^{4}$ Zuyan Xu, ${ }^{7}$ Chuangtian Chen, ${ }^{7}$ and X. J. Zhou ${ }^{1,2,8,}$,

${ }^{1}$ Beijing National Laboratory for Condensed Matter Physics, Institute of Physics, Chinese Academy of Sciences, Beijing 100190, China

${ }^{2}$ University of Chinese Academy of Sciences, Beijing 100049, China

${ }^{3}$ Max Planck Institute of Microstructure Physics, Weinberg 2, Halle 06120, Germany

${ }^{4}$ Hefei National Laboratory for Physical Science at Microscale and Department of Physics, University of Science and Technology of China, Hefei 230026, China

${ }^{5}$ Insitute of Theoretical Physics and Astrophysics, Julius-Maximilaians University of Wurzburg, Am Hubland, Wurzburg D-97074, Germany

${ }^{6}$ Military Transportation University, Tianjin 300161, China

${ }^{7}$ Technical Institute of Physics and Chemistry, Chinese Academy of Sciences, Beijing 100190, China

${ }^{8}$ Collaborative Innovation Center of Quantum Matter, Beijing 100871, China

(Received 3 May 2018; published 2 August 2018)

\begin{abstract}
The iron-based superconductors are characterized by multiple-orbital physics where all the five Fe $3 d$ orbitals get involved. The multiple-orbital nature gives rise to various novel phenomena like orbitalselective Mott transition, nematicity, and orbital fluctuation that provide a new route for realizing superconductivity. The complexity of multiple-orbital physics also requires us to disentangle the relationship between orbital, spin, and nematicity, and to identify dominant orbital ingredients that dictate superconductivity. The bulk FeSe superconductor provides an ideal platform to address these issues because of its simple crystal structure and unique coexistence of superconductivity and nematicity. However, the orbital nature of the low-energy electronic excitations and its relation to the superconducting gap remain controversial. Here, we report direct observation of the highly anisotropic Fermi surface and extremely anisotropic superconducting gap in the nematic state of the FeSe superconductor by highresolution laser-based angle-resolved photoemission measurements. We find that the low-energy excitations of the entire hole pocket at the Brillouin zone center are dominated by the single $d_{x z}$ orbital. The superconducting gap exhibits an anticorrelation relation with the $d_{x z}$ spectral weight near the Fermi level; i.e., the gap size minimum (maximum) corresponds to the maximum (minimum) of the $d_{x z}$ spectral weight along the Fermi surface. These observations provide new insights in understanding the orbital origin of the extremely anisotropic superconducting gap in the FeSe superconductor and the relation between nematicity and superconductivity in the iron-based superconductors.
\end{abstract}

DOI: 10.1103/PhysRevX.8.031033

In the iron-based superconductors, all the five $\mathrm{Fe} 3 d$ orbitals $\left(d_{x z}, d_{y z}, d_{x y}, d_{x^{2}-y^{2}}\right.$, and $\left.d_{z^{2}}\right)$ are involved in the lowenergy electronic excitations [1,2]. The multiple-orbital

\footnotetext{
* Corresponding author. XJZhou@iphy.ac.cn

Published by the American Physical Society under the terms of the Creative Commons Attribution 4.0 International license. Further distribution of this work must maintain attribution to the author(s) and the published article's title, journal citation, and DOI.
}

Subject Areas: Condensed Matter Physics, Superconductivity

character provides a new degree of freedom which, when combined with charge and spin, brings new phenomena like orbital-selective Mott transition [3,4], orbital ordering [5,6], and nematicity [7]. Orbital fluctuation may provide a new channel for realizing superconductivity $[8,9]$. On the other hand, such multiple-orbital nature also brings complexity in finding the key ingredients of superconductivity in the ironbased superconductors. FeSe is unique in the iron-based superconductors because it has the simplest crystal structure [10]. It shows a nematic transition at $\sim 90 \mathrm{~K}$ without being accompanied by a magnetic transition [11]. In particular, the FeSe superconductor provides an ideal case for studying the 
(a)

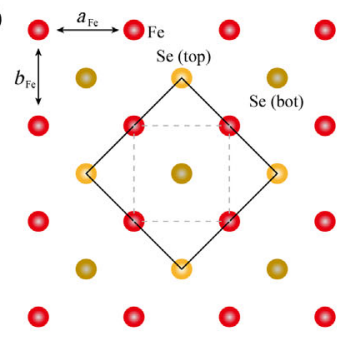

(b)

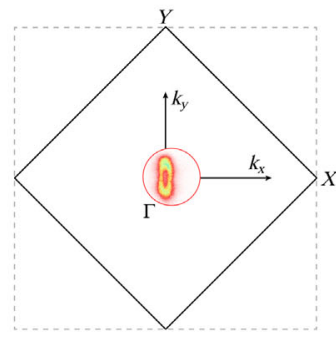

(c)

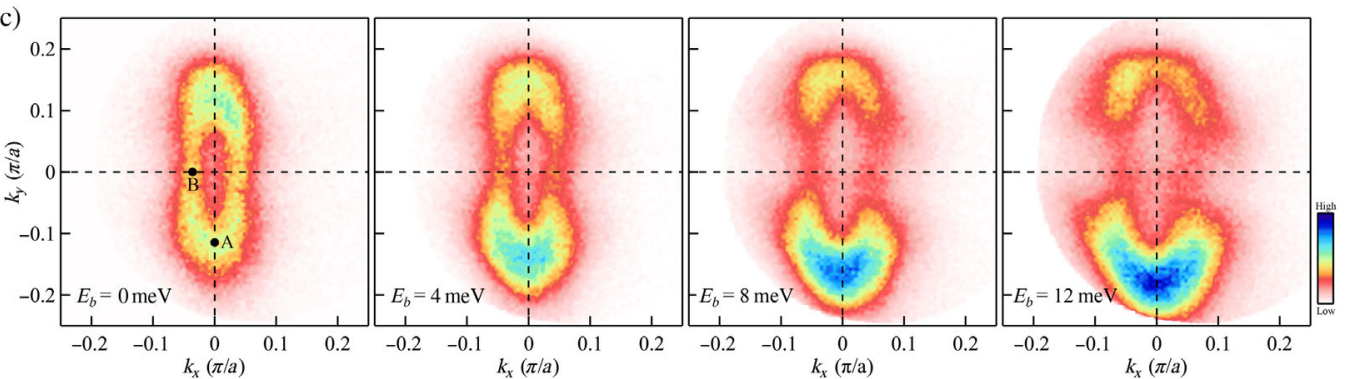

(d)

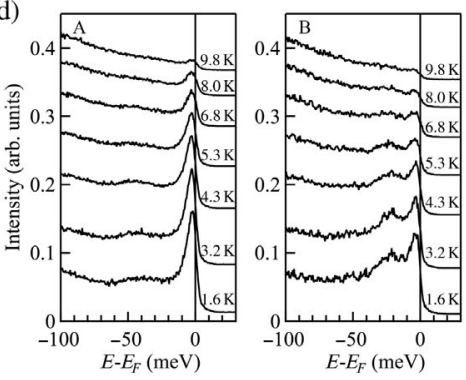

(e)

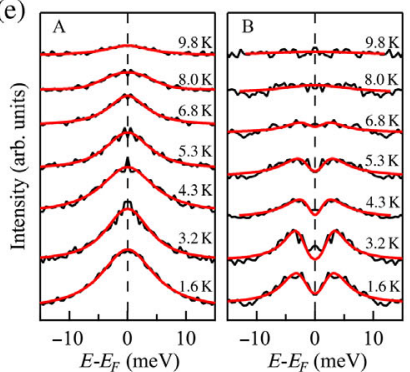

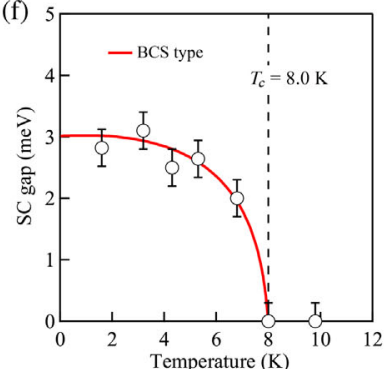

FIG. 1. Fermi surface of the single domain FeSe measured at $1.6 \mathrm{~K}$ around the $\Gamma$ point and its superconducting gap. (a) Top view of FeSe crystal structure. The red, yellow, and gray circles represent Fe atom, Se atom above the Fe plane (Se top), and Se atom below the plane (Se bottom), respectively. The dashed line represents the 1-Fe unit cell while the solid line represents the 2-Fe unit cell. We define $x$ axis parallel to $a_{\mathrm{Fe}}$ axis and $y$ axis parallel to $b_{\mathrm{Fe}}$ axis. In the normal state, FeSe has $C_{4}$ symmetry with $a_{\mathrm{Fe}}=b_{\mathrm{Fe}}$, but it breaks into $C_{2}$ symmetry in the nematic state with $a_{\mathrm{Fe}}>b_{\mathrm{Fe}}$. (b) The Brillouin zone of FeSe. The dashed line and the solid line correspond to BZs of the 1-Fe unit cell and 2-Fe unit cell, respectively. The red circle represents the momentum area that can be measured simultaneously by our laser ARPES system with $6.994 \mathrm{eV}$ photon energy. The whole Fermi surface of FeSe around the $\Gamma$ point can be covered by one time measurement which occupies a very small portion of the BZ. (c) Constant energy contours around the $\Gamma$ point at different binding energies measured at $1.6 \mathrm{~K}$ in $L V$ polarization geometry. The measured Fermi surface (leftmost panel) shows an elliptical shape with its long axis along $k_{y}$ axis and short axis along $k_{x}$ axis. The spectral weight concentrates to the vertex areas and gets depleted around the central area with increasing binding energy. (d) Photoemission spectra (EDCs) measured at different temperatures for two typical Fermi momenta. The locations of the two momenta, A and B, are marked in (c) as black dots. (e) Corresponding symmetrized EDCs from (d). The curves are fitted with a phenomenological gap formula [41] (solid red curves). For point A (left-hand panel), no gap opening is detected below $T_{c}$ within our experimental resolution. Apparent gap opening occurs for point B (right-hand panel). (f) Temperature dependence of the superconducting gap at B point. It follows the BCS gap form (red curve).

relationship between nematicity and superconductivity [12-15] because superconductivity occurs in the nematic state. However, the experimental results are controversial regarding the superconducting gap of FeSe on whether it is nodeless [16-24] or nodal [25-27]. It also remains highly controversial on the orbital nature of the low-energy excitations that dictates superconductivity [22-24,27-32]. Direct determination of the correspondence between the orbital nature of the low-energy electronic states and the superconducting gap is crucial to understand the superconductivity mechanism of the iron-based superconductors.

In this paper, we performed high-resolution laser-based angle-resolved photoemission (ARPES) measurements on the electronic structure and superconducting gap of the bulk FeSe superconductor $\left(T_{c}=8.0 \mathrm{~K}\right)$ in the nematic state. The highly anisotropic Fermi surface around the Brillouin zone (BZ) center is observed with the aspect ratio of $\sim 3$ between the long axis (along $k_{y}$ ) and short axis (along $k_{x}$ ). The superconducting gap along the Fermi pocket is extremely anisotropic, varying between $\sim 3 \mathrm{meV}$ along the short axis of the Fermi surface to zero along the long axis within our experimental precision $( \pm 0.2 \mathrm{meV})$. Detailed band structure analysis, combined with band structure calculations, indicates that the Fermi surface is dominated by a single $d_{x z}$ orbital. Moreover, we find that the superconducting gap size shows an anticorrelation with the $d_{x z}$ spectral weight near the Fermi level; the gap minimum (maximum) corresponds to the $d_{x z}$ spectral intensity maximum (minimum) along the Fermi surface. These observations provide key insights on the orbital origin of the anisotropic electronic structure and superconducting gap in FeSe and the interplay between nematicity and superconductivity in the iron-based superconductors.

The electronic structure and superconducting gap of the FeSe superconductor $\left(T_{c}=8.0 \mathrm{~K}\right.$ ) (see Methods below and Fig. S1 of Supplemental Material [33] for sample details) were measured by a high-resolution laser-based angleresolved photoemission system based on the time-of-flight electron energy analyzer (see Methods and Fig. S2 [33] for experimental details). This new ARPES system has an advantage of covering two-dimensional momentum space at the same time with high-energy resolution $(\sim 1 \mathrm{meV})$ 
and momentum resolution. It is also equipped with an ultralow-temperature cryostat which can cool down the sample to $1.6 \mathrm{~K}$. The laser polarization can be tuned to identify the orbital character of the observed band structure by taking advantage of the photoemission matrix element effects [34]. This system is particularly suitable for bulk FeSe because of its low $T_{c}$, tiny Fermi pockets, and very small superconducting gap.

The FeSe sample we measured has a structural phase transition $\mathrm{T}_{s}$ around $90 \mathrm{~K}$ [Fig. S1(b) of Supplemental Material [33] ]. It assumes a tetragonal crystal structure above $T_{s}$ and becomes orthorhombic below $T_{s}$, where the distance between adjacent $\mathrm{Fe}$ is slightly different [Fig. 1(a), where $a_{\mathrm{Fe}}\left(b_{\mathrm{Fe}}\right)$ is defined as the long $x$ (short $y$ ) axis]. Dramatic anisotropy of physical properties between $a_{\mathrm{Fe}}$ and $b_{\mathrm{Fe}}$ axes occurs below the nematic transition temperature [35-38] although the lattice constant difference between $a_{\mathrm{Fe}}$ and $b_{\mathrm{Fe}}$ is only $\sim 0.5 \%$ [39]. Figure 1(b) shows the measured Fermi surface of FeSe around the BZ center which occupies a small portion of the entire BZ. Our laserbased ARPES system made it possible to cover the whole Fermi pocket around the $\Gamma$ point simultaneously under the same condition with very dense momentum points. Figure 1(c) shows constant energy contours of FeSe at different binding energies measured at $1.6 \mathrm{~K}$. The measured Fermi surface [leftmost panel of Fig. 1(c)] is highly anisotropic: the Fermi momentum along the $k_{x}$ direction is $\sim 0.036 \pi / a$ while it is $\sim 0.11 \pi / a$ along the $k_{y}$ direction, resulting in an elongated ellipse with a high aspect ratio of $\sim 3$. This is the Fermi surface that exhibits the strongest anisotropy observed among all the iron-based superconductors [40]. For the photon energy we used $(6.994 \mathrm{eV})$, the measured Fermi surface corresponds to a $k_{z}$ close to zero [30]. With increasing binding energy, the constant energy contours increase in area [Fig. 1(c)], consistent with the Fermi pocket being holelike. In the meantime, the spectral weight is gradually concentrated onto the two vertex areas along the long axis while it gets strongly suppressed in the central region. We note that for the FeSe single crystal sample we measured here, it has nearly pure single domain even though we did not detwin the sample in advance. This may be due to accidental internal stress exerted in the sample during the preparation process because in most cases we can see signals from the coexisting two domains (Fig. S2 [33]). In the FeSe sample we measured (Fig. 1), the signal from another domain is negligible even under different light polarizations. The single domain FeSe sample provided us an ideal opportunity to study its intrinsic electronic structure and superconducting gap, as we present below.

Figure 1(d) shows photoemission spectra [energy distribution curves (EDCs)] of FeSe measured at two typical momentum points along the Fermi surface $[\mathrm{A}$ and $\mathrm{B}$ points as marked in Fig. 1(c)] at different temperatures. The corresponding symmetrized EDCs are shown in Fig. 1(e). When the sample cools down from the normal state to the superconducting state, for both momentum points A and B, sharp superconducting coherence peaks develop which get stronger with decreasing temperature [Fig. 1(d)]. The sharp coherence peak with a width of $\sim 6 \mathrm{meV}$ is observed at $1.6 \mathrm{~K}$. The EDC symmetrization is a standard procedure to remove the Fermi-Dirac function in order to extract the superconducting gap [41]. The gap size can be determined by the distance between the two peaks in the symmetrized EDCs which can be fitted by a phenomenological gap formula [41]. For point A, the symmetrized EDCs always show a peak at the Fermi level [left-hand panel in Fig. 1(e)], indicating no detectable gap opening in the superconducting state within our experimental resolution. On the other hand, for point B, the symmetrized EDCs clearly show two peaks in the superconducting state [right-hand panel in Fig. 1(e)] indicative of a superconducting gap opening. The extracted superconducting gap at different temperatures [Fig. 1(f)] follows a BCS gap form; it becomes zero at and above the superconducting transition temperature of $8.0 \mathrm{~K}$.

Figure 2 shows detailed momentum dependence of the superconducting gap for FeSe measured at $1.6 \mathrm{~K}$. The symmetrized EDCs at some representative Fermi momenta are shown in Fig. 2(a). Figure 2(b) is a false-color image which represents the same data as in Fig. 2(a). The location of the Fermi momenta is defined by the Fermi surface angle $\theta$, as indicated in Fig. 2(c). As seen directly from Figs. 2(a) and 2(b), the superconducting gap shows a maximum near $\theta=0^{\circ}$ and $180^{\circ}$ and becomes rather small, basically zero within our experimental precision $( \pm 0.2 \mathrm{meV})$, near $\theta=90^{\circ}$ and $270^{\circ}$. This is consistent with the recent result that the superconducting gap approaches minima near the $\theta=90^{\circ}$ region although their gap measurement covers a small portion of the Fermi surface in detwinned FeSe $[23,24,27]$. The symmetrized EDCs are fitted by the phenomenological gap formula, as shown in Fig. 2(a), and the extracted superconducting gap is plotted in Fig. 2(d) as a function of the Fermi surface angle. The simultaneous twodimensional momentum coverage of our laser ARPES system makes it possible to have very dense data points of the superconducting gap along the entire Fermi surface measured with superhigh resolution. This allows us to quantitatively examine various possible gap functions. The twofold symmetry of the superconducting gap has excluded the possibility of a pure $s$-wave which would have fourfold symmetry. The twofold symmetry, together with two nearzero points, is rather reminiscent of a $p$-wave gap form. The measured gap is fitted by the $p$-wave form $\Delta_{p}=\left|\Delta_{0} \cos (\theta)\right|$ and the fitted curve is shown as a green line in Fig. 2(d). An alternative gap form is $s+d$ type, which can also assume twofold symmetry $[42,43]$. In Fig. 2(d), we also fitted the measured data with two types of $s+d$ form. We first tried $\Delta_{s, d}=\left|\Delta_{0}+\Delta_{1} \cos (2 \theta)\right|$, which contains a simple $s$-wave form $\Delta_{0}$ and a $d$-wave form $\Delta_{1} \cos (2 \theta)$. The fitted curve [blue line in Fig. 2(d)] exhibits an obvious deviation from the measured data, particularly near the minimal and maximal 

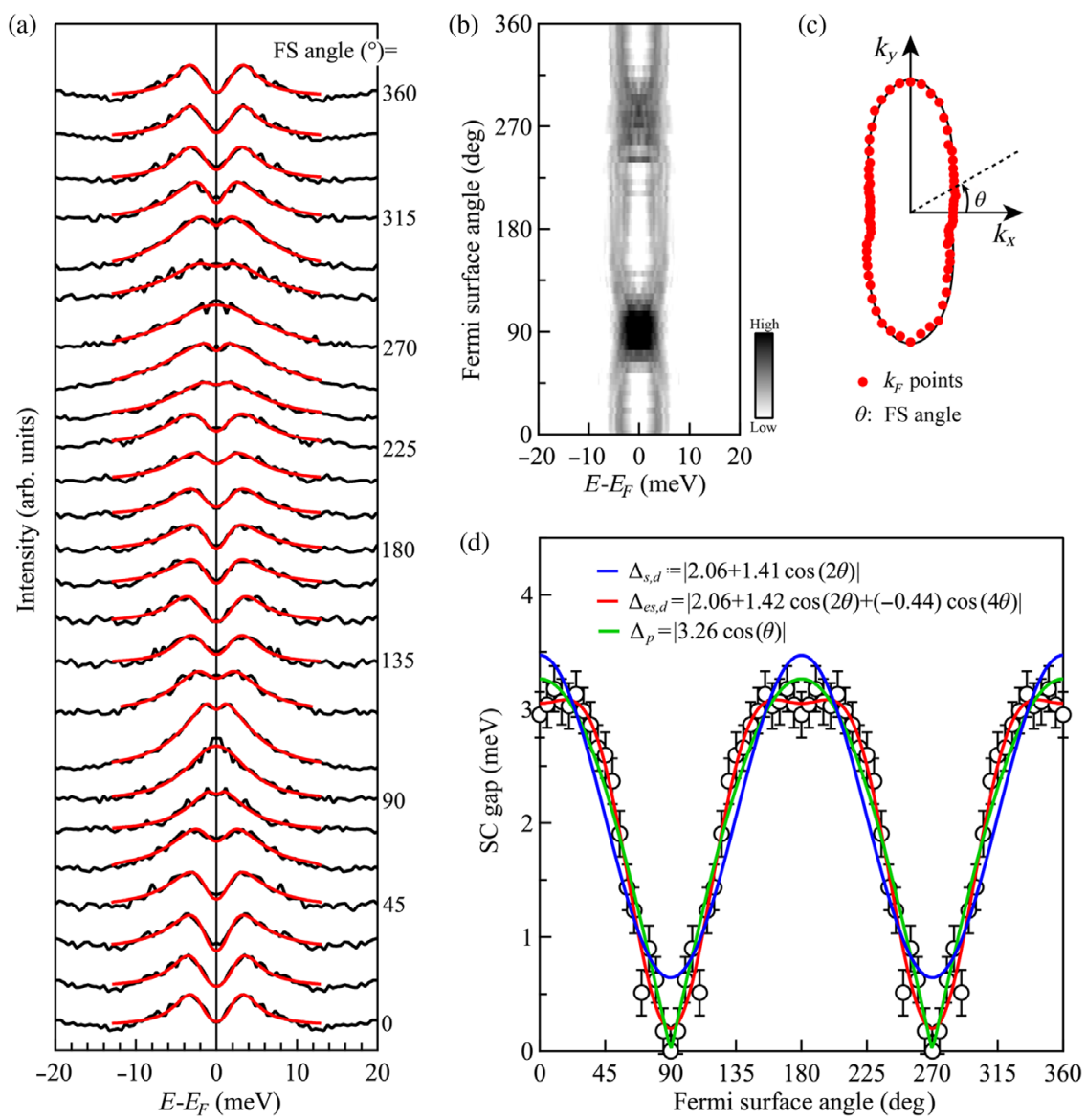

FIG. 2. Momentum dependence of the superconducting gap for FeSe measured at 1.6 K. (a) Symmetrized EDCs at different Fermi momenta along the Fermi surface measured at $1.6 \mathrm{~K}$. These curves are fitted with a phenomenological gap formula (red curves). The same data are plotted in (b) as a false-color image where the variation of the superconducting peak position can be directly visualized. The location of the Femi momentum is defined by the Fermi surface (FS) angle $\theta$ as shown in (c). (d) Momentum dependence of the superconducting gap derived from fitting the symmetrized EDCs in (a). To enhance the data statistics and keep the twofold symmetry, the gap value is obtained by averaging over the four quadrants. The measured gap (empty circles) is fitted by $s+d$ (blue curve), extended $s+d$ (red curve), and $p$-wave (green curve) pairing gap forms.

gap regions. Then we tried $\Delta_{e s, d}=\mid \Delta_{0}+\Delta_{1} \cos (2 \theta)+$ $\Delta_{2} \cos (4 \theta) \mid$, which contains an anisotropic $s$-wave $\Delta_{0}+$ $\Delta_{2} \cos (4 \theta)$ and a $d$-wave form $\Delta_{1} \cos (2 \theta)$. The fitted curve is marked as a red line in Fig. 2(d). We note that within our experimental precision $( \pm 0.2 \mathrm{meV})$, we can not differentiate between the cases of zero node, two nodes, and four nodes along the Fermi surface for the $e s+d$ gap form (Fig. S6 [33]). The observation of two nodes on the Fermi surface in terms of the $e s+d$ gap form is quite accidental because three fitting parameters are needed and a constraint has to be imposed between these parameters; i.e., $\left(\Delta_{0}+\Delta_{2}\right)-\Delta_{1}=0$ [Fig. S6(e) [33] ]. Our precise measurement on the momentum dependence of the superconducting gap puts a strong constraint on the possible gap form in the FeSe superconductor.

Figure 3 shows detailed momentum dependence of the band structure for FeSe measured at $1.6 \mathrm{~K}$, along the momentum cuts crossing the BZ center with different
Fermi surface angles defined as $\theta$ in Fig. 3(a). Two main bands are observed for all the momentum cuts varying from horizontal $\left(\theta=0^{\circ}\right)$ to vertical $\left(\theta=90^{\circ}\right)$ directions, labeled as $\alpha$ and $\beta$ bands shown in the leftmost and rightmost panels of Fig. 3(b). When the momentum cuts vary from vertical $\left(\theta=90^{\circ}\right)$ to horizontal $\left(\theta=0^{\circ}\right)$ directions, the Fermi momentum of the $\alpha$ band significantly decreases and the band gets steeper. The top of this $\alpha$ band lies at $\sim 6.7 \mathrm{meV}$ above the Fermi level, as estimated by fitting the holelike band with a parabola [red curve in the leftmost panel in Fig. 3(b)]. The top of the $\beta$ band stays at $\sim 20 \mathrm{meV}$ below the Fermi level [dashed green line in the leftmost panel in Fig. 3(b)]. This band gets flatter when the momentum cuts change from vertical $\left(\theta=90^{\circ}\right)$ to horizontal $\left(\theta=0^{\circ}\right)$ directions. Based on the analysis of the photoemission matrix elements (see Fig. S2 of Supplemental Material [33]), the $\alpha$ and $\beta$ bands can be attributed to the $d_{x z}$ and $d_{y z}$ orbitals, respectively. This assignment is further supported 

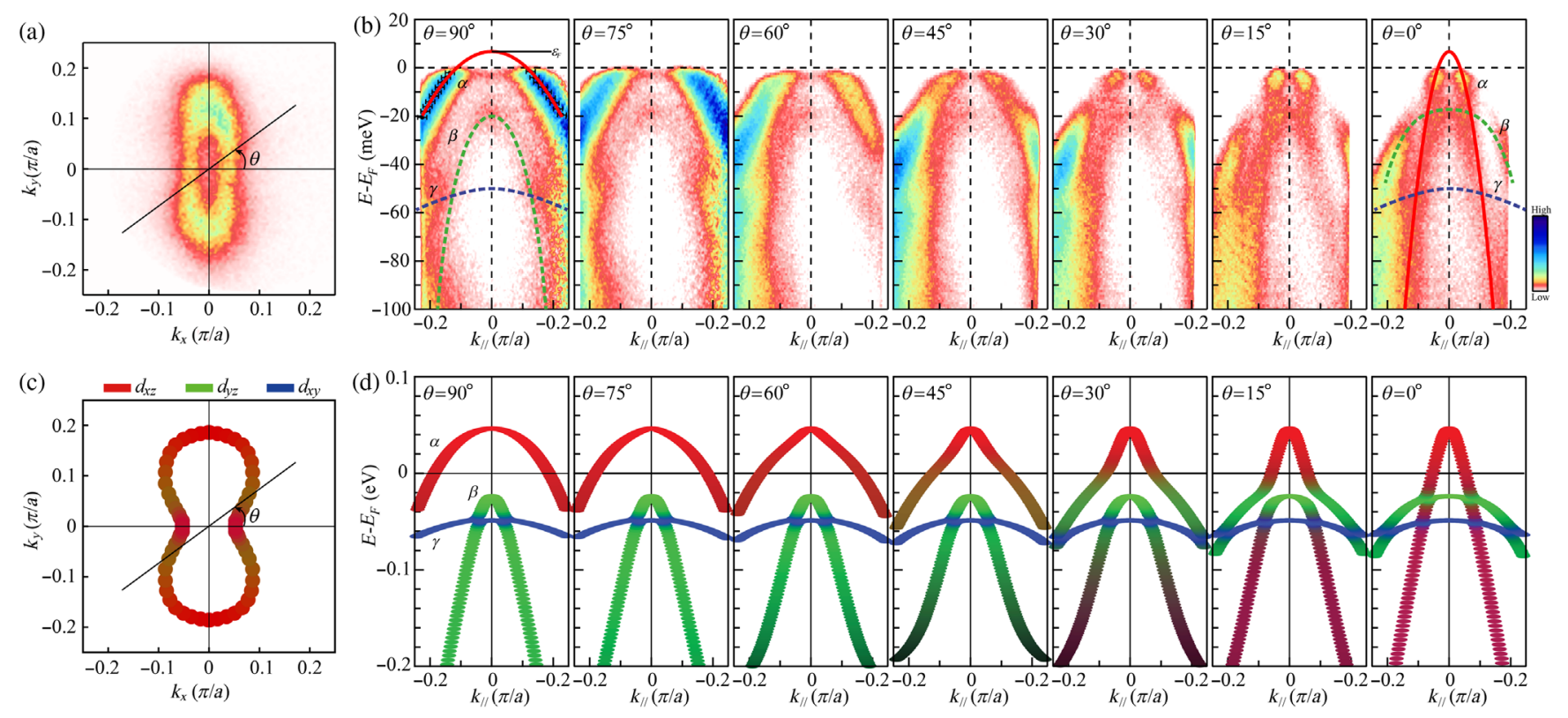

FIG. 3. Band structure of FeSe along different momentum cuts and their orbital nature. (a) Definition of the momentum cuts across the $\Gamma$ point by the Fermi surface angle $\theta$. (b) Measured band structure along different momentum cuts varying from $\theta=90^{\circ}$ (vertical cut, leftmost panel) to $\theta=0^{\circ}$ (horizontal cut, rightmost panel). Three bands are observed, marked as $\alpha, \beta$, and $\gamma$ in the $\theta=90^{\circ}$ and $\theta=0^{\circ}$ panels. The $\alpha$ band is well fitted by a parabolic curve (red line) which gives a Fermi energy $\varepsilon_{F}$ of $\sim 6.7$ meV above the Fermi level. The effective mass for different momentum cuts can be obtained from such a parabolic fitting. The dashed green and blue lines are guides to the eye for the $\beta$ and $\gamma$ bands, respectively. (c) The calculated Fermi surface of FeSe around $\Gamma$ point in the nematic state. (d) The calculated band structure along different momentum cuts defined by the Fermi surface angle $\theta$ in (c). In panels (c) and (d), the $d_{x z}, d_{y z}$, and $d_{x y}$ orbital components are represented by red, green, and blue colors, respectively.

by our band structure calculations [Figs. 3(c) and 3(d)], which capture the momentum dependence of the two bands well [see Methods (below) and Supplemental Material [33] ]. Above the nematic transition, $d_{x z}$ and $d_{y z}$ bands are split because of the spin-orbital coupling [Figs. S3(a) and S3(b) [33] ] [30]. In the nematic state, the $d_{x z}$ band is pushed up while the $d_{y z}$ band is pushed down below the Fermi level [Figs. S3(c) and S3(d) [33]] [28], resulting in only one holelike Fermi pocket around $\Gamma$, which is dominated by the $d_{x z}$ orbital. In particular, the electronic states at the Fermi level along the $k_{x}$ and $k_{y}$ axes are purely from the $d_{x z}$ orbitals, as demonstrated by the matrix elements analysis (Fig. S2 [33]) and the band structure calculations [Figs. 3(c) and 3(d)]. We note that there is a slight band hybridization of $d_{x z}$ and $d_{y z}$ orbitals on some portions of the Fermi surface with a Fermi surface angle between $\theta=15^{\circ}-45^{\circ}$ [Figs. 3(c) and 3(d)]. But even in this case, the $d_{x z}$ orbital still dominates the electronic states at the Fermi level. There has been controversy regarding the orbital nature of the electronic states near the Fermi level $[22,28,30,31]$. Our results demonstrate unambiguously that it is the $d_{x z}$ orbital that dominates the low-energy electronic structure of FeSe around the $\Gamma$ point in the nematic state.

After resolving the dominant $d_{x z}$ orbital nature that dictates the Fermi surface and superconducting gap of the FeSe superconductor, now we investigate some key factors that are closely related to its superconductivity. Figure 4(a) shows the momentum distribution curves
(MDCs) at the Fermi level along various Fermi surface angles $\theta$ [schematically shown in the top left-hand inset in Fig. 4(a)] measured in the normal state at $9.8 \mathrm{~K}$. The spectral weight [red region in Fig. 4(a)] is strongest near $\theta=90^{\circ}$ and gets weaker when moving to $\theta=0^{\circ}$ and $180^{\circ}$. Quantitative analysis of the spectral weight (MDC area) as a function of the Fermi surface angle is plotted in Fig. 4(d); it is peaked near $\theta=90^{\circ}$ and $270^{\circ}$. Figure 4(b) shows photoemission spectra (EDCs) along the Fermi surface measured at $9.8 \mathrm{~K}$ in the normal state, and the spectral weight of the $d_{x z}$ orbital [red region in Fig. 4(b)] at different Fermi surface angles is plotted in Fig. 4(e). It shows similar variation with the MDC weight. The analysis in the superconducting state gives similar results (Fig. S4 [33]). For comparison, the superconducting gap of FeSe as a function of the Fermi surface angle measured at $1.6 \mathrm{~K}$ is replotted in Fig. 4(c). Overall, the superconducting gap exhibits an anticorrelation relation with the spectral weight of the $d_{x z}$ orbital; i.e., the gap minimum corresponds to the spectral weight maximum. We also analyzed the effective mass of the $\alpha$ band as the function of the Fermi surface angle, as shown in Fig. 4(f). The effective mass displays a dramatic variation along the Fermi surface; its value along the $k_{x}$ axis is nearly 10 times that along the $k_{y}$ axis. It also shows a perfect anticorrelation with the superconducting gap: the gap maximum (minimum) corresponds to the minimum (maximum) of the effective mass. 

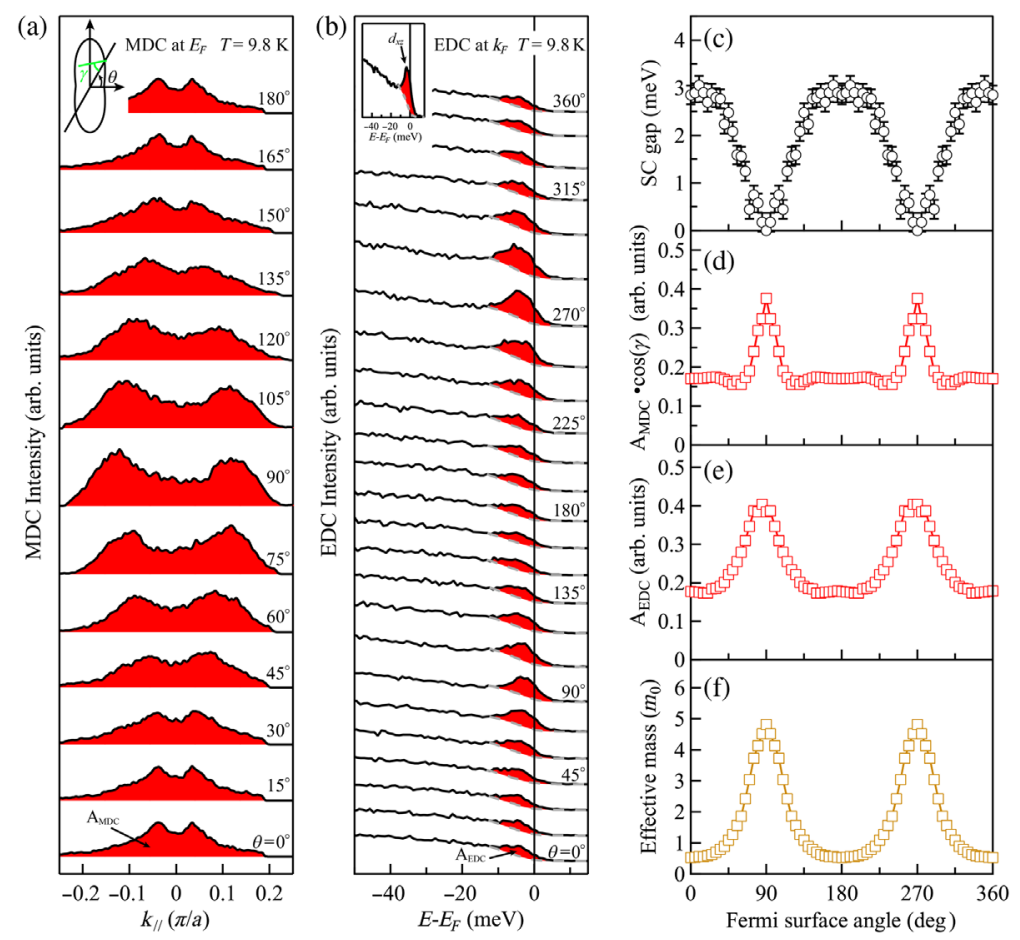

(g)

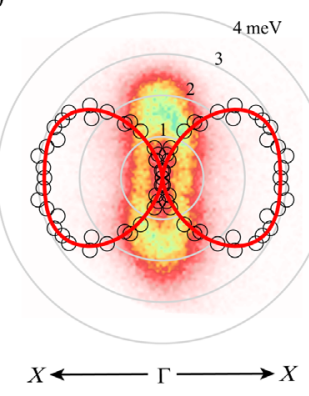

(h)

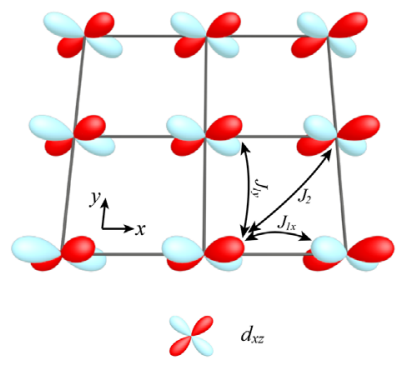

FIG. 4. Correlation between the superconducting gap, the spectral weight, and the effective mass of bands along the Fermi surface of FeSe. (a) Momentum distribution curves (MDCs) along different momentum cuts at the Fermi level measured at $9.8 \mathrm{~K}$. The momentum cut is defined by the Fermi surface angle $\theta$ as shown in the inset. For a given Fermi momentum on the Fermi surface, the angle between the momentum cut and the Fermi surface normal direction is defined as an angle $\gamma$. The area of the MDCs marked in red is defined as the spectral weight $A_{\mathrm{MDC}}$ in (d). (b) Photoemission spectra (EDCs) of FeSe along the Fermi surface measured at $9.8 \mathrm{~K}$. After subtracting the Shirley background (dashed line), the area of the EDCs marked in red is defined as the spectral weight $A_{\mathrm{EDC}}$ in (e). For the convenience of comparison, the superconducting gap measured at $1.6 \mathrm{~K}$ is replotted in (c). (d) MDC spectral weight along the Fermi surface obtained from (a) after considering the correction of the $\gamma$ angle as defined in inset of (a). (e) EDC spectral weight along the Fermi surface obtained from (b). (f) Effective mass of the $d_{x z}$ band along different momentum cuts as obtained by a parabolic fitting of the $\alpha$ band in Fig. 3(b). (g) Superconducting gap plotted in the polar graph, overlaid with the measured Fermi surface in order to have a direct comparison of their relative orientations. (h) A schematic picture of the $d_{x z}$ orbitals in the Fe plane in the nematic state of FeSe. For the $d_{x z}$ orbitals, the dominant intraorbital coupling is the exchange interactions along the $x$ axis $\left(J_{1 x}\right)$. Both $J_{1 y}$ and $J_{2}$ are antiferromagneticlike while the $J_{1 x}$ coupling is ferromagnetic in iron chalcogenides [44-47].

The present observations provide key information on understanding the relationship between nematicity, electronic structure, and superconductivity in FeSe. In the nematic state, the splitting of the $d_{x z}$ and $d_{y z}$ orbitals gives rise to an anisotropic Fermi surface around the $\Gamma$ and $X / Y$ points (corresponding to the $\mathrm{BZ}$ of a $1-\mathrm{Fe}$ unit cell) [30,31]. In the extreme case when the low-energy electronic states are dominated by a single $d_{x z}$ orbital, the electron hopping along the $x$ direction is much more enhanced than that along the $y$ direction [Fig. 4(h)], resulting in highly anisotropic Fermi surface topology [Fig. 1(c)] and effective mass [Fig. 4(f)] in the nematic state of FeSe. Since a nearly isotropic superconducting gap with fourfold symmetry is observed in tetragonal $\mathrm{Fe}(\mathrm{Se}, \mathrm{Te})$ superconductors $[48,49]$, the observed extremely anisotropic superconducting gap with twofold symmetry in FeSe is expected to be associated with its nematicity. The question arises as to how nematicity can generate such an anisotropic superconducting gap. One scenario proposed involves the splitting of the $d_{x z}$ and $d_{y z}$ orbitals $[42,50]$. The orbital order in the nematic state introduces a $d$-wave component on top of the existing $s$-wave component, leading to a highly anisotropic superconducting gap with even accidental nodes [42]. Our measured superconducting gap [Fig. 2(d)] is consistent with the expected $e s+d$-type gap form in this picture [42]. However, it is expected that the superconducting gap is positively correlated with the orbital occupation [50], which is opposite to our observation that the gap and the spectral weight exhibit an anticorrelation [Figs. 4(c)-4(e)]. This picture involves two hole pockets around the $\Gamma$ point, each of which is composed of both $d_{x z}$ and $d_{y z}$ orbitals $[42,50]$. It needs to be modified in order to make a direct comparison with our results of FeSe where there is only one Fermi pocket around the $\Gamma$ point which is dominated by a single $d_{x z}$ orbital.

To understand the anisotropic superconducting gap of $\mathrm{FeSe}$, another scenario proposed considers orbitalselective Cooper pairing in a modified spin-fluctuation 
theory via suppression of pair scattering processes involving those less coherent states [22]. This picture can produce a superconducting gap structure that is consistent with our results [Fig. 2(d)] and previous experimental observations $[22,43]$. It was proposed that the pairing is mainly based on electrons from the $d_{y z}$ orbital of the iron atoms [22,29]. However, the scanning tunneling microscope (STM) or scanning tunneling spectroscopy (STS) measurements [22] cannot provide direct information on the orbital nature of the Fermi surface. Our observations provide direct evidence that, in the nematic state of FeSe, the extremely anisotropic superconducting gap opens on the highly anisotropic Fermi surface [Fig. 4(g)] that is dominated by the $d_{x z}$ orbital. In $\mathrm{FeSe}$, it was also proposed that the superconducting gap shows a positive correlation with the spectral weight of the $d_{y z}$ orbital that is considered to be responsible for superconductivity [22]. From our direct ARPES results, although we find that the spectral weight of the $d_{y z}$ orbital exhibits a good correspondence to the superconducting gap (Fig. S5 [33]), the $\beta$ band with $d_{y z}$ orbital character lies $\sim 20 \mathrm{meV}$ below the Fermi level, thus contributing little to superconductivity of FeSe. Our results indicate that superconductivity in $\mathrm{FeSe}$ is dictated by the $d_{x z}$ orbital and its spectral weight shows an anticorrelation with the superconducting gap: the gap minimum corresponds to the spectral weight maximum of the $d_{x z}$ orbital [Figs. 4(c)4(e)]. Our results ask for a reexamination of the picture of the orbital-selective superconductivity in FeSe [22]. A recent work [23] also found that the central hole pocket consists mainly of the $d_{x z}$ orbital that is consistent with our results. However, they stated that the superconducting gap follows positively with the spectral weight from the $d_{y z}$ orbital, despite the fact that the $d_{y z}$ orbital composes only a small portion of the spectral weight [23]. We note that such an assignment of the dominant orbital that dictates superconductivity on the central hole pocket and the relation between the superconducting gap and the spectral weight contradict our results.

The fact that the measured gap function can be fitted to a simple $p$-wave gap function also leads us to ask whether the spin-triplet pairing is possible in FeSe. In the nematic state, because of the dominant single $d_{x z}$ orbital feature of the Fermi surface around the $\Gamma$ and the strong Hund's rule coupling, the interaction of the $d_{x z}$ band with other bands near $X / Y$ is expected to become rather weak. For the $d_{x z}$ orbital, the dominant intraorbital coupling is the nearestneighbor magnetic exchange couplings along the $x$ axis, namely the $J_{1 x}$ interaction shown in Fig. 4(h), which is ferromagnetic in iron chalcogenides from neutron scattering measurements [44-47]. If this is the strongest pairing interaction, it is possible for the superconducting pairing on the Fermi surface to have a triplet $p$-wave symmetry with a gap function proportional to $\cos (\theta)$, which is consistent with our observations. No drop of the Knight shift across
$T_{c}$ is observed in NMR measurements of bulk FeSe, which is also compatible with a possible triplet pairing [37,38]. In addition to the coexisting Néel and stripe spin fluctuations observed by neutron scattering [51,52], recent observation of charge ordering in FeSe suggests the presence of an additional magnetic fluctuation with a rather small wave vector [53] that is related to intrapocket scattering around the $\Gamma$ point [54]. Our present work raises an interesting possibility of $p$-wave pairing symmetry in FeSe. We note that ARPES can measure only the magnitude of the superconducting order parameter. Also, because of the limitation of the laser photon energy $(6.994 \mathrm{eV})$, it is not possible for us to measure the superconducting gap on the Fermi surface around the $X / Y$ point [Fig. 1(b)]. Considering that the measured superconducting gap can be fitted quite well by both a simple $p$-wave form [green line in Fig. 2(d)] and a combined gap form of anisotropic $s$ and $d$ waves [red line in Fig. 2(d)], it is clear that further experimental and theoretical studies are needed to establish the pairing symmetry of FeSe.

In summary, by carrying out high-resolution laser-based ARPES on the bulk FeSe, we have observed an extremely anisotropic superconducting gap on the highly anisotropic Fermi surface in the nematic state of FeSe superconductor. The Fermi pocket around $\Gamma$ is dominated by $d_{x z}$ orbital. The spectral weight of the $d_{x z}$ orbital shows an anticorrelation with the superconducting gap along the Fermi surface. Our results directly demonstrate that the Cooper pairing on the Fermi pocket around $\Gamma$ originates from electrons of $d_{x z}$ orbitals in the nematic state of FeSe. They provide key insights on the pairing symmetry and the interplay between nematicity and superconductivity in iron-based superconductors.

Methods. - High-quality FeSe bulk single crystals were grown by the $\mathrm{KCl} / \mathrm{AlCl}_{3}$ chemical vapor transport technique $[55,56]$. The samples are characterized by $\mathrm{x}$-ray diffraction [Fig. S1(a) [33] ]. Electrical resistance measurement and magnetic measurement [Figs. S1(b) and S1(c) [33] ] show a $T_{c}$ of $8.0 \mathrm{~K}$ with a sharp transition width of $\sim 0.4 \mathrm{~K}$.

High-resolution ARPES measurements were performed at our new laser-based system equipped with a $6.994-\mathrm{eV}$ vacuum ultraviolet laser and a time-of-flight electron energy analyzer (ARToF 10k by Scienta Omicron) [57]. This latest-generation ARPES system is capable of measuring photoelectrons covering two-dimensional momentum space $\left(k_{x}, k_{y}\right)$ simultaneously. The system is equipped with an ultralow-temperature cryostat which can cool the sample to a low temperature of $1.6 \mathrm{~K}$. Measurements were performed using both $L H$ and $L V$ polarization geometries (see Fig. S2 [33]). The energy resolution is $\sim 1 \mathrm{meV}$ and the angular resolution is $\sim 0.1^{\circ}$. All the samples were measured in ultrahigh vacuum with a base pressure better than $5.0 \times 10^{-11}$ mbar. The samples were cleaved in situ and measured at different temperatures. The Fermi level is referenced by measuring polycrystalline gold, which is in 
good electrical contact with the sample, as well as the normal state measurement of the sample above $T_{c}$.

To simulate the band structure of FeSe, we adopted the five-orbital tight-binding model $[58,59]$ including on-site spin-orbital coupling $(\lambda)$. In the nematic state, we further consider $s$-wave $\left(\Delta_{s}\right)$ and $d$-wave $\left(\Delta_{d}\right)$ orbital order which break $C_{4}$ rotational symmetry. The Hamiltonian of these two orders is given by

$$
\begin{aligned}
& H_{s}=\sum_{\boldsymbol{k}} \Delta_{s}\left(\cos k_{x}+\cos k_{y}\right)\left(n_{x z, \boldsymbol{k}}-n_{y z, \boldsymbol{k}}\right), \\
& H_{d}=\sum_{\boldsymbol{k}} \Delta_{d}\left(\cos k_{x}-\cos k_{y}\right)\left(n_{x z, \boldsymbol{k}}+n_{y z, \boldsymbol{k}}\right),
\end{aligned}
$$

where $n_{\alpha, k}=n_{\alpha, k \uparrow}+n_{\alpha, k \downarrow}$ is the density for the $\alpha$ orbital. To match the data in ARPES experiments in the nematic state, the adopted parameters in the calculations are $\lambda=10 \mathrm{meV}, \Delta_{s}=17.5 \mathrm{meV}$, and $\Delta_{d}=-10 \mathrm{meV}$. In the normal state, we set $\lambda=10 \mathrm{meV}, \Delta_{s}=0 \mathrm{meV}$, and $\Delta_{d}=0 \mathrm{meV}$. Here, the strength of the spin-orbital coupling $(\lambda=10 \mathrm{meV})$ is determined by the splitting of the $d_{y z}$ and $d_{x z}$ orbital above $T_{s}$ from Ref. [30].

\section{ACKNOWLEDGMENTS}

We thank Dunghai Lee, Rafael Fernandes, Chandra Varma, Qimiao Si, Guangming Zhang, J.C. Seamus Davis, Zhixun Shen, and Guoqing Zheng for helpful discussions. This work is supported by the National Key Research and Development Program of China (Grants No. 2016YFA0300300 and No. 2017YFA0302900), the National Natural Science Foundation of China (Grants No. 11334010 and No. 11534007), the National Basic Research Program of China (Grants No. 2015CB921000 and No. 2015CB921300), and the Strategic Priority Research Program (B) of the Chinese Academy of Sciences (Grants No. XDB07020300 and No. XDPB01).

D. L., C. L., and J. Huang contributed equally to this work. X. J. Z. and D. L. proposed and designed the research. B. L., L. W., Y. S., and X. C. contributed to FeSe crystal growth. Y.M. and X. D. contributed to the magnetic measurement. X. W., J. Hu, and T.X. contributed to the band structure calculations and theoretical discussion. D. L., C. L., J. Huang, B. S., Q. G., Y. Z., X. L., Y. H., Y. X., A. L., J. L., P. A., L. Z., S. H., L. Y., G. L., F. Z., S. Z., F. Y., Z. W., Q. P., Z.X., C. C., and X. J.Z. contributed to the development and maintenance of Laser-ARTOF system. X. J. contributed to software development for data analysis. D. L., C. L., J. Huang, and B. S. carried out the experiment with assistance from Q. G., Y. Z., X. L., Y. H., Y. X., A. L., J. L., and P. A. Additionally, D. L., C. L., J. Huang, and X. J.Z. analyzed the data. D. L. and X. J. Z. wrote the paper with C. L., J. Huang, J. Hu, and T. X. All authors participated in discussions and comments on the paper.

The authors declare no competing financial interests.
[1] S. Lebegue, Electronic Structure and Properties of the Fermi Surface of the Superconductor LaOFeP, Phys. Rev. B 75, 035110 (2007).

[2] J. Paglione and R. L. Greene, High-Temperature Superconductivity in Iron-Based Materials, Nat. Phys. 6, 645 (2010).

[3] M. Yi, D. Lu, R. Yu, S. Riggs, J.-H. Chu, B. Lv, Z. Liu, M. Lu, Y.-T. Cui, M. Hashimoto, S.-K. Mo, Z. Hussain, C. Chu, I. Fisher, Q. Si, and Z.-X. Shen, Observation of Temperature-Induced Crossover to an Orbital Selective Mott Phase in $\mathrm{A}_{x} \mathrm{Fe}_{2-y} \mathrm{Se}_{2}(\mathrm{~A}=\mathrm{K}, R b)$ Superconductors, Phys. Rev. Lett. 110, 067003 (2013).

[4] R. Yu and Q. Si, Orbital-Selective Mott Phase in Multiorbital Models for Alkaline Iron Selenides $\mathrm{K}_{1-x} \mathrm{Fe}_{2-y} \mathrm{Se}_{2}$, Phys. Rev. Lett. 110, 146402 (2013).

[5] F. Kruger, S. Kumar, J. Zaanen, and J. Brink, Spin-Orbital Frustrations and Anomalous Metallic State in Iron-Pnictide Superconductors, Phys. Rev. B 79, 054504 (2009).

[6] C.-C. Lee, W.-G. Yin, and W. Ku, Ferro-orbital Order and Strong Magnetic Anisotropy in the Parent Compounds of Iron-Pnictide Superconductors, Phys. Rev. Lett. 103, 267001 (2009).

[7] R. Fernandes, A. V. Chubukov, and J. Schmalian, What Drives Nematic Order in Iron-Based Superconductors?, Nat. Phys. 10, 97 (2014).

[8] T. Saito, S. Onari, and H. Kontani, Orbital Fluctuation Theory in Iron Pnictides: Effects of $\mathrm{As}-\mathrm{Fe}-\mathrm{As}$ Bond Angle, Isotope Substitution, and $Z^{2}$-Orbital Pocket on Superconductivity, Phys. Rev. B 82, 144510 (2010).

[9] H. Kontani and S. Onari, Orbital-Fluctuation-Mediated Superconductivity in Iron Pnictides Analysis of the FiveOrbital Hubbard-Holstein Model, Phys. Rev. Lett. 104, 157001 (2010).

[10] F. Hsu, J. Luo, K. Yeh, T. Chen, T. Huang, P. M. Wu, Y. Lee, Y. Huang, Y. Chu, D. Yan, and M. Wu, Superconductivity in the PbO-Type Structure $\alpha$-FeSe, Proc. Natl. Acad. Sci. U.S.A. 105, 14262 (2008).

[11] T. McQueen, Q. Huang, V. Ksenofontov, C. Felser, Q. Xu, H. Zandbergen, Y. Hor, J. Allred, A. J. Williams, D. Qu, J. Checkelsky, N. P. Ong, and R. J. Cava, Extreme Sensitivity of Superconductivity to Stoichiometry in $\mathrm{Fe}_{1+\delta} \mathrm{Se}$, Phys. Rev. B 79, 014522 (2009).

[12] F. Wang, S. A. Kivelson, and D.-H. Lee, Nematicity and Quantum Paramagnetism in FeSe, Nat. Phys. 11, 959 (2015).

[13] J. K. Glasbrenner, I. I. Mazin, H. O. Jeschke, P. J. Hirschfeld, R. M. Fernandes, and R. Valenti, Effect of Magnetic Frustration on Nematicity and Superconductivity in Iron Chalcogenides, Nat. Phys. 11, 953 (2015).

[14] R. Yu and Q. Si, Antiferroquadrupolar and Ising-Nematic Orders of a Frustrated Bilinear-Biquadratic Heisenberg Model and Implications for the Magnetism of FeSe, Phys. Rev. Lett. 115, 116401 (2015).

[15] X. Wu, Y. Liang, H. Fan, and J. Hu, Nematic Orders and Nematicity-Driven Topological Phase Transition in FeSe, arXiv:1603.02055.

[16] J. Dong, T. Guan, S. Zhou, X. Qiu, L. Ding, C. Zhang, U. Patel, Z. Xiao, and S. Y. Li, Multigap Nodeless Superconductivity in $\mathrm{FeSe}_{x}$ : Evidence from Quasiparticle Heat Transport, Phys. Rev. B 80, 024518 (2009). 
[17] P. Bourgeois-Hope, S. Chi, D. Bonn, R. Liang, W. Hardy, T. Wolf. C. Meingast, N. Doiron-Leyraud, and L. Taillefer, Thermal Conductivity of the Iron-Based Superconductor FeSe: Nodeless Gap with a Strong Two-Band Character, Phys. Rev. Lett. 117, 097003 (2016).

[18] J. Lin, Y. Hsieh, D. Chareev, A. Vasiliev, Y. Parsons, and H. Yang, Coexistence of Isotropic and Extended S-Wave Order Parameters in FeSe as Revealed by Low-Temperature Specific Heat, Phys. Rev. B 84, 220507 (2011).

[19] L. Jiao, C.-L. Huang, S. Robler, C. Koz, U. K. Robler, U. Schwarz, and S. Wirth, Superconducting Gap Structure of FeSe, Sci. Rep. 7, 44024 (2017).

[20] M. Abdel-Hafiez, J. Ge, A. Vasiliev, D. Chareev, J. Vondel, V. V. Moshchalkov, and A. V. Silhanek, Temperature Dependence of Lower Critical Field $\mathrm{H}_{c 1}(T)$ Shows Nodeless Superconductivity in FeSe, Phys. Rev. B 88, 174512 (2013).

[21] R. Khasanov, M. Bendele, A. Amato, K. Conder, H. Keller, H.-H. Klause, H. Luetkens, and E. Pomjakushina, Evolution of Two-Gap Behavior of the Superconductor $\mathrm{FeSe}_{1-x}$, Phys. Rev. Lett. 104, 087004 (2010).

[22] P. Sprau, A. Kostin, A. Kreisel, A. E. Bohmer, V. Taufour, P. C. Canfield, S. Mukherjee, P. J. Hirschfeld, B. M. Andersen, and J. C. Davis, Discovery of Orbital-Selective Cooper Pairing in FeSe, Science 357, 75 (2017).

[23] L. C. Rhodes, M. D. Watson, A. A. Haghighirad, D. V. Evtushinsky, M. Eschrig, and T. K. Kim, Scaling of the Superconducting Gap with Orbital Character in FeSe, arXiv: 1804.01436.

[24] Y. S. Kushnirenko, A. V. Fedorov, E. Haubold, S. Thirupathaiah, T. Wolf, S. Aswartham, I. Morozov, T. K. Kim, B. Buchner, and S. V. Borisenko, Three-Dimensional Superconducting Gap in FeSe from Angle-Resolved Photoemission Spectroscopy, Phys. Rev. B 97, 180501(R) (2018).

[25] C.-L. Song, Y.-L. Wang, P. Cheng, Y.-P. Jiang, W. Li, T. Zhang, Z. Li, K. He, L. Wang, J.-F. Jia, H.-H. Hung, C. Wu, X. Ma, X. Chen, and Q.-K. Xue, Direct Observation of Nodes and Twofold Symmetry in FeSe Superconductor, Science 332, 1410 (2011).

[26] S. Kasahara, T. Watashige, T. Hanaguri, Y. Kohsaka, T. Yamashita, Y. Shimoyama, Y. Mizukami, R. Endo, H. Ikeda, K. Aoyama, T. Terashima, S. Uji, T. Wolf, H. Lohneysen, T. Shibauchi, and Y. Matsuda, Field-Induced Superconducting Phase of FeSe in the BCS-BEC Cross-Over, Proc. Natl. Acad. Sci. U.S.A. 111, 16309 (2014).

[27] T. Hashimoto, Y. Ota, H. Q. Yamamoto, Y. Suzuki, T. Shimojima, S. Watanabe, C. Chen, S. Kasahara, Y. Matsuda, T. Shibauchi, K. Okazaki, and S. Shin, Superconducting Gap Anisotropy Sensitive to Nematic Domains in FeSe, Nat. Commun. 9, 282 (2018).

[28] Y. Suzuki, T. Shimojima, T. Sonobe, A. Nakamura, M. Sakano, H. Tsuji, J. Omachi, K. Yoshioka, M. KuwataGonokami, T. Watashige, R. Kobayashi, S. Kasahara, T. Shibauchi, Y. Matsuda, Y. Yamakawa, H. Kontani, and K. Ishizaka, Momentum-Dependent Sign Inversion of Orbital Order in Superconducting FeSe, Phys. Rev. B 92, 205117 (2015).

[29] A. Kreisel, B. M. Andersen, P. O. Sprau, A. Kostin, J. C. Davis, and P. J. Hirschfeld, Orbital Selective Pairing and
Gap Structures of Iron-Based Superconductors, Phys. Rev. B 95, 174504 (2017).

[30] M. Watson, T. Kim, A. Haghighirad, N. Davies, A. McCollam, A. Narayanan, S. F. Blake, Y. Chen, S. Ghannadzadeh, A. Schofield, M. Hoesch, C. Meingast, T. Wolf, and A. I. Coldea, Emergence of the Nematic Electronic State in FeSe, Phys. Rev. B 91, 155106 (2015).

[31] M. Watson, T. Kim, L. Rhodes, M. Eschrig, M. Hoesch, A. Haghighirad, and A. I. Coldea, Evidence for Unidirectional Nematic Bond Ordering in FeSe, Phys. Rev. B 94, 201107(R) (2016).

[32] M. Watson, A. Haghighirad, L. Rhodes, M. Hoesch, and T. Kim, Electronic Anisotropies Revealed by Detwinned Angle-Resolved Photoemission Spectroscopy Measurements of FeSe, New J. Phys. 19, 103021 (2017).

[33] See Supplemental Material at http://link.aps.org/ supplemental/10.1103/PhysRevX.8.031033 for details on the characterization of FeSe crystal, the orbital composition analysis of the central hole pocket, the band structure calculations, the momentum-dependent spectral weight analysis of $d_{x z}$ and $d_{y z}$ orbitals in the superconducting state, the analysis of the measured superconducting gap.

[34] A. Damascelli, Z. Hussain, and Z.-X. Shen, Angle-Resolved Photoemission Studies of the Cuprate Superconductors, Rev. Mod. Phys. 75, 473 (2003).

[35] M. Tanatar, A. Bohmer, E. I. Timmons, M. Schutt, G. Drachuck, V. Taufour, K. Kothapalli, A. Kreyssig, S. Budko, P. C. Canfield, R. M. Fernandes, and R. Prozorov, Origin of the Resistivity Anisotropy in the Nematic Phase of FeSe, Phys. Rev. Lett. 117, 127001 (2016).

[36] A. Boehmer and A. Kreisel, Nematicity, Magnetism and Superconductivity in FeSe, J. Phys. Condens. Matter 30, 023001 (2018).

[37] S. Baek, D. Efremov, J. Ok, J. Kim, J. Brink, and B. Buchner, Orbital-Driven Nematicity in FeSe, Nat. Mater. 14, 210 (2015).

[38] A. Boehmer, T. Arai, F. Hardy, T. Hattori, T. Iye, T. Wolf, H. Lohneysen, K. Ishida, and C. Meingast, Origin of the Tetragonal-to-Orthorhombic Phase Transition in FeSe: A Combined Thermodynamic and NMR Study of Nematicity, Phys. Rev. Lett. 114, 027001 (2015).

[39] S. Margadonna, Y. Takabayashi, M. T. McDonald, K. Kasperkiewicz, Y. Mizuguchi, Y. Takano, A. N. Fitch, E. Suard, and K. Prassides, Crystal Structure of the New $\mathrm{FeSe}_{1-x}$ Superconductor, Chem. Commun. (Cambridge) 0, 5607 (2008).

[40] D. Liu et al., Common Electronic Features and Electronic Nematicity in Parent Compounds of Iron-Based Superconductors and $\mathrm{FeSe} / \mathrm{SrTiO}_{3}$ Films Revealed by AngleResolved Photoemission Spectroscopy, Chin. Phys. Lett. 33, 077402 (2016).

[41] M. Norman, M. Randeria, H. Ding, and J. C. Campuzano, Phenomenology of the Low-Energy Spectral Function in High $-\mathrm{T}_{c}$ Superconductors, Phys. Rev. B 57, 11093(R) (1998).

[42] J. Kang, A. F. Kemper, and R. M. Fernandes, Manipulation of Gap Nodes by Uniaxial Strain in Iron Based Superconductors, Phys. Rev. Lett. 113, 217001 (2014).

[43] H. Xu, X. Niu, D. Xu, J. Jiang, Q. Yao, Q. Chen, Q. Song, M. Abdel-Hafiez, D. Chareev, A. Vasiliev, Q. Wang, H. Wo, 
J. Zhao, R. Peng, and D. L. Feng, Highly Anisotropic and Twofold Symmetric Superconducting Gap in Nematically Ordered $\mathrm{FeSe}_{0.93} \mathrm{~S}_{0.07}$, Phys. Rev. Lett. 117, 157003 (2016).

[44] D. Johnston, The Puzzle of High Temperature Superconductivity in Layered Iron Pnictides and Chalcogenides, Adv. Phys. 59, 803 (2010).

[45] J. Hu, B. Xu, W. Liu, N. Hao, and Y. Wang, Unified Minimum Effective Model of Magnetic Properties of IronBased Superconductors, Phys. Rev. B 85, 144403 (2012).

[46] M. Wang, C. Fang, D. Yao, G. Tan, L. W. Harriger, Y. Song, T. Netherton, C. Zhang, M. Wang, M. B. Stone, W. Tian, J. $\mathrm{Hu}$, and P. Dai, Spin Waves and Magnetic Exchange Interactions in Insulating $\mathrm{Rb}_{0.89} \mathrm{Fe}_{1.58} \mathrm{Se}_{2}$, Nat. Commun. 2, 580 (2011).

[47] P. Dai, Antiferromagnetic Order and Spin Dynamics in Iron-Based Superconductors, Rev. Mod. Phys. 87, 855 (2015).

[48] K. Okazaki, Y. Ito, Y. Kotani, T. Shimojima, T. Kiss, S. Watanabe, C.-T. Chen, S. Nitaka, T. Hanaguri, H. Takagi, A. Chainani, and S. Shin, Evidence for a $\cos (4 \varphi)$ Modulation of the Superconducting Energy Gap of Optimally Doped $\mathrm{FeTe}_{0.6} \mathrm{Se}_{0.4}$ Single Crystals Using Laser Angle-Resolved Photoemission Spectroscopy, Phys. Rev. Lett. 109, 237011 (2012).

[49] H. Miao, P. Richard, Y. Tanaka, K. Nakayama, T. Qian, K. Umezawa, T. Sato, Y. Xu, Y. Shi, N. Xu, X. Wang, P. Zhang, H. Yang, Z. Xu, J. Wen, G.-D. Gu, X. Dai, J. Hu, T. Takahashi, and H. Ding, Isotropic Superconducting Gaps with Enhanced Pairing on Electron Fermi Surfaces in $\mathrm{FeTe}_{0.55} \mathrm{Se}_{0.45}$, Phys. Rev. B 85, 094506 (2012).

[50] T. Agatsuma and H. Yamase, Structure of the Pairing Gap from Orbital Nematic Fluctuations, Phys. Rev. B 94, 214505 (2016).

[51] Q. Wang, Y. Shen, B. Pan, Y. Hao, M. Ma, F. Zhou, P. Steffens, K. Schmalzl, T. R. Forrest, A. Hafiez, X. Chen,
D. Chareev, A. Vasiliev, P. Bourges, Y. Sidis, H. Cao, and J. Zhao, Strong Interplay between Stripe Spin Fluctuations, Nematicity and Superconductivity in FeSe, Nat. Mater. 15, 159 (2016).

[52] Q. Wang, Y. Shen, B. Pan, X. Zhang, K. Ikeuchi, K. Iida, A. Christianson, H. Walker, D. Adroja, M. Hafiez, X. Chen, D. Chareev, A. Vasiliev, and J. Zhao, Magnetic Ground State of $\mathrm{FeSe}$, Nat. Mater. 7, 12182 (2016).

[53] W. Li, Y. Zhang, P. Deng, Z. Xu, S.-K. Mo, M. Yi, H. Ding, M. Hashimoto, R. G. Moore, D. Lu, X. Chen, Z.-X. Shen, and Q.-K. Xue, Stripes Developed at the Strong Limit of Nematicity in FeSe Film, Nat. Phys. 13, 957 (2017).

[54] Y. Tam, D. Yao, and W. Ku, Itinerancy-Enhanced Quantum Fluctuation of Magnetic Moments in Iron-Based Superconductors, Phys. Rev. Lett. 115, 117001 (2015).

[55] B. Lei, J. Cui, Z. Xiang, C. Shang, N. Wang, G. Ye, X. Luo, T. Wu, Z. Xun, and X.H. Chen, Evolution of HighTemperature Superconductivity from Low- $\mathrm{T}_{c}$ Phase Tuned by Carrier Concentration in FeSe Thin Flakes, Phys. Rev. Lett. 116, 077002 (2016).

[56] A. Boehmer, F. Hardy, F. Eilers, D. Ernst, P. Adelmann, P. Schweiss, T. Wolf, and C. Meingast, Lack of Coupling between Superconductivity and Orthorhombic Distortion in Stoichiometric Single-Crystalline FeSe, Phys. Rev. B 87, 180505(R) (2013).

[57] Y. Zhang et al., Electronic Evidence of Temperature Induced Lifshitz. Transition and Topological Nature in $\mathrm{ZrTe}_{5}$, Nat. Commun. 8, 15512 (2017).

[58] Y. Su, H. Liao, and T. Li, The Form and Origin of Orbital Ordering in the Electronic Nematic Phase of Iron-Based Superconductors, J. Phys. Condens. Matter 27, 105702 (2015).

[59] T. Li and Y. Su, Driving Force of the Orbital-Relevant Electronic Nematicity in Fe-Based Superconductors, J. Phys. Condens. Matter 29, 425603 (2017). 